\title{
A Globally-Implicit Computational Framework for Physics-based Simulation of Coupled Thermo-Hydro-Mechanical Problems: Application to Sustainability of Geothermal Reservoirs
}

\author{
Robert Podgorney ${ }^{1}$, Hai Huang, Mitch Plummer, Derek Gaston \\ ${ }^{1}$ Idaho National Laboratory, 2525 Fremont Ave, Idaho Falls, ID 83415
}

Simulation Notes Europe SNE 23(1), 2013, 51 - 58

DOI: $10.11128 /$ sne.23.tn. 10175

Received: Feb. 10, 2013 (Selected SIMS 2012 Postconf. Publ.);

Accepted: March 20, 2013;

Abstract. This paper highlights the development of a fully-coupled and fully-implicit modeling tool for predicting the dynamics of fluid flow, heat transport, and rock deformation using a GIA named FALCON (Fracturing And Liquid CONvection). The code is developed on a parallel Multiphysics Object Oriented Simulation Environment (MOOSE) computational framework developed at Idaho National Laboratory (INL) for providing finite element solutions of coupled system of nonlinear partial differential equations. In this paper, a brief overview of the governing equations numerical approach are discussed, and an example simulation of strongly coupled geothermal reservoir behavior is presented.

\section{Introduction}

Numerical modeling has played an important role in understanding the behavior of geothermal systems since as early as the 1970s. While capabilities of geothermal reservoir simulators have grown since then, the prospect of simulating more challenging classes of geothermal problems-such as reservoir creation and operation of engineered geothermal systems (EGS), high enthalpy supercritical magmatic systems, etc-pose additional, and very significant, computational challenges that the current generation of continuum or dual-continuum hydrothermal models are ill-equipped to describe.
Interest in multiphysics simulation techniques is growing rapidly with a focus on more realistic and higher fidelity analysis of geothermal and engineering systems. This in- crease in activity is typically attributed to increasing computer power and more robust computational schemes $[9,11,12]$, but in truth, advanced numerical methods are playing an equal role. The phrase 'multiphysics simulation' is used to describe analyses which include disparate physical phenomena-such as coupled multiphase, multicomponent fluid flow, enthalpy transport, and geomechanics and their feedbacks are examined in a simultaneous manner. Examples of multiphysics problems in subsurface energy applications are numerous and include pressure and temperature driven permeability creation and evolution in geothermal reservoirs, temperature driven phase evolution of in-situ kerogen processing of oil shale reservoirs, kinetically controlled reactive transport in the flow of contaminants, etc. In addition to multiphysics coupling, most of these problems also have multiscale issues to resolve.

Examining coupled physics for fluid flow, energy transport, and geomechanical deformation is a relatively new area for the geothermal community; however, simulating coupled problems has been an important topic of study in the reactive transport community for decades. Yeh and Tripathi [21] and Steefel and MacQuarrie [16] cite three major approaches that differ in the way coupling transport and reaction have been considered for reactive transport modeling: (1) GIA (fully-coupled) approach that solves all governing nonlinear equations simultaneously at each time step using various forms of Newton's method, (2) sequential iteration approach 
(SIA) that subdivide the reactive transport problem into transport and reaction subproblems, solves them sequentially, and then iterates, and (3) sequential noniteration approach (SNIA) that solves the transport and reaction problems sequentially without iteration, which is often referred as operator-splitting. The operatorsplitting approach is perhaps the simplest to implement and requires the least computational resources in terms of the memory and CPU time; thus, it became the method of choice for subsurface reactive transport modeling during the past three decades.

However, the drawback of the operator-splitting approach is the splitting error when the physics (either reactions-transport or flow-mechanics) are tightly coupled; the solution becomes inaccurate and requires very small time steps [17]. For most potential EGS reservoirs fluid flow, heat transport, and rock deformation will be strongly nonlinearly coupled. The changes in flow and energy transport properties due to fracturing and/or dissolution add further complexity and nonlinearity to the problem. For such situations, the global implicit approach (GIA) solves all solution variables simultaneously during each time step by seeking the solution of a large system of nonlinear equations via some form of Newton's method and is a more robust solution than the other two approaches [3, 8, 14].

One potential limitation of the GIA approach is the need to compute, store and invert the Jacobian matrix. This could become problematic for large systems which would be expected for reservoir-scale geothermal problems. As the number of solution variables grows, the matrix holding the Jacobian entries also grows. The increased size of the Jacobian matrix results in greater memory usage and more CPU time to solve the resulting system of linear equations within the Newton iterations. For highly nonlinear processes involving strong fluid-reservoir interactions and significant changes of flow and transport properties due to fracturing, the true Jacobian is often difficult to describe in analytical formulas. For reasons such as these, during the past three decades, despite its numerical merits of greater robustness and the ability to take larger time steps, the fullycoupled GIA method was considered to be too CPUtime and memory-intensive [21] or to be computationally inefficient [16]. It has been used primarily only as a research tool for small one- or two-dimensional problems with a few thousands of unknowns. Since the first attempts of implementing the GIA approach in the early 1980s [17, 13], only a handful of examples based on this approach have been reported in the literature, compared with numerous examples of applications based on an operator splitting approach [19, 20, 15].

\section{Architecture and Design}

FALCON has been designed for the simulation of geothermal reservoirs, both conventional hydrothermal and EGS. The architecture of FALCON has a plug-and-play modular design structure based on representing each piece of the residual term in a weak form of the governing PDEs as a 'Kernel'. Kernels may be coupled together to achieve different application goals. All kernels are required to supply a residual, which usually involves summing products of finite element shape functions. The basic architecture of the code allows convenient coupling of different processes and incorporation of new physics.

Figure 1 shows the basic architecture of FALCON, with the Kernels at the uppermost level, directly underlain by the numerical framework and solver libraries used to couple the Kernels and perform reservoir simulations. Currently primary Kernels (primary variables) have been written to describe the following physics:

- Single-phase flow of water

- Two-phase flow of water and steam

- Conservative heat transport

- Enthalpy transport

- Fluid and Energy Sources/Sinks

- Displacement (all mechanics are solved in terms of displacement)

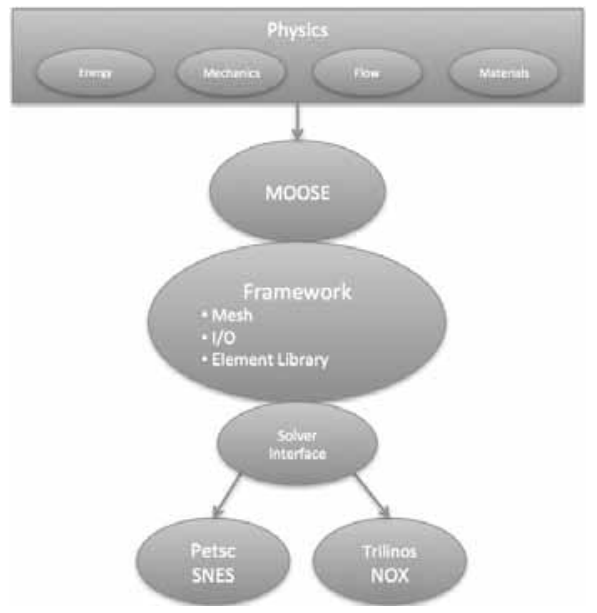

Figure 1: Kernel and Object Oriented Architecture used to develop the FALCON simulator. 
For any given simulation, any combination of the primary kernels can be applied to make the problem as simple or complex as necessary, with some exceptions. Single phase flow of water problems must be cast in terms of temperature, while steam-water flow problems must use enthalpy for energy transport. The option of single phase temperature formulation was the basis for early versions of FALCON and have been retained because of their computational and memory efficiency. As an example of the modular framework, one can simply choose only a single phase pressure kernel, and solve a simple LaPlace equation based on imposed boundary conditions, totally ignoring energy and mechanics kernels.

An auxiliary variable system has been built into FALCON to handle solving most all of the derived quantities and variables that are dependent on the primary kernels mentioned above. The number of auxiliary kernels needed for a given simulation depends on the choice of primary variables and whether they are formulated in terms of pressure-temperature or pressureenthalpy. In general, a simulation run with the pressureenthalpy formulation, considering geomechanical displacement and damaging, requires the most auxiliary kernels and has the highest computational burden. The auxiliary kernels consist of

- Equation of state calculations

- Steam and water density

- Steam and water viscosity

- Derivatives of steam and water density to pressure, temperature, or enthalpy as required

- Stress and Strain

- Fluid Velocities

- Damage Menchanics (or fracturing)

In addition to the primary and auxiliary physics kernels, other kernels are required for the mesh, material properties (and some additional supporting calculations), boundary conditions, code execution/solver parameters, and data output.

\subsection{Code uses and limitations}

As stated above, the FALCON code has been developed to support simulation of both conventional hydrothermal and EGS reservoirs, with a primary design focus on EGS resources. While we are using the IAPWS-97 formulation [18], which has an quite an effective operating range of pressure $(\leq 100 \mathrm{MPa})$ and temperature
( $\leq 800 \mathrm{C})$. Code development to date has focused on subcritical conditions.

Maximum mesh sizes are related to the number of kernels, and hence the total system wide Degrees of Freedom (DoFs), used in a simulation. In practice, the true limitations are based on computational power and available memory. The parallel scaling and performance example testing used 1 million grid blocks and more than 20 million DoFs, and showed remarkable scalability. Code tests have used computational meshes with greater than 30 million elements and also showed excellent scaling performance [7]. For any parallel simulation runs, a minimum of 20,000 DoFs per processor is recommended for good scalability.

\section{Numerical Methodology}

FALCON has been developed using INL's MOOSE framework [6]. This framework provides a strong numerical foundation for rapid development of multidimensional, parallel, fully implicit, fully-coupled, nonlinear simulation capabilities. MOOSE is based on a finite element discretization strategy and utilizes stateof-the-art preconditioned Jacobian-Free Newton-Krylov (JFNK) nonlinear solution method that requires only residual evaluations of the discrete system. Strategic use of this feature results in a modular, pluggable architecture that greatly simplifies adding new physics and coupling them together. The MOOSE framework incorporates multiple parallel solution capabilities including both Message Passing Interface (MPI) and threading utilizing the Intel Threading Building Blocks (TBB), which allows application codes developed upon MOOSE to run efficiently on multicore workstations, laptops and supercomputers. All parallel activities are completely hidden from application developers, enabling scientists and engineers to focus on the physics of problem they wish to solve instead of parallel programing practices.

In addition, applications developed upon MOOSE also inherit many advanced computing capabilities such as dimension-independence, massive paral- lelism, high-order finite elements and adaptive mesh refinement/coarsening with both structured and unstructured meshes. 


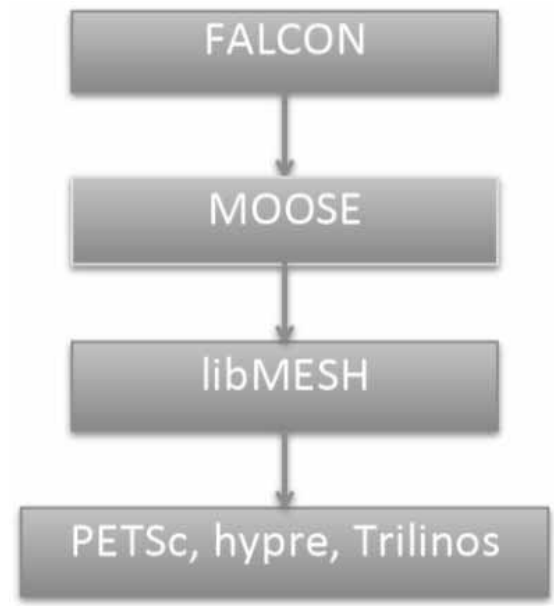

Figure 2: Hierarchical framework used to build the FALCON simulator, based up the INL developed MOOSE library [6]. The libMesh finite element framework developed by the CFDLab at the University of Texas at Austin [34] provides a core set of parallel finite-element libraries and couples with interfaces to linear and nonlinear solvers from both Petsc [2] and Trilinos [9] along with other packages such as Hypre [4].

The MOOSE framework has a layered structure, as shown in Figure 2. The lower layer interfaces with several open-source libraries from multiple universities and national laboratories. In particular, the libMesh finite element framework developed by the CFDLab at the University of Texas at Austin [10] provides a core set of parallel finite-element libraries. Coupled with interfaces to linear and nonlinear solvers from both PETSc [2] and Trilinos [9] along with other packages such as Hypre [4], MOOSE and application codes developed upon it provide considerable flexibility including the abilities to swap out solver libraries and to utilize diverse large scale parallel computing resources.

The middle layer of MOOSE provides a set of core functionalities necessary for residual and Jacobian (more precisely, the preconditioner) evaluations required by the preconditioned JFNK approach, such as fetching the designated test and shape functions, numerical integration using Gaussian quadrature, and coupling physics. The top layer of MOOSE, referred as the kernel is the interface with physics where the FALCON application is built (see Figure 2). It is convenient to think of a kernel as a piece of the residual term in the weak forms of PDEs, for example, the diffusion term, advection term, time accumulation term in the weak form of general enthalpy transport equations. Kernels may be coupled together to achieve different application goals. All kernels are required to supply a residual, which usually involves summing products of finite element shape functions.

Kernels may also provide diagonal and off-diagonal blocks of the (approximate) Jacobian matrix for the purpose of building certain preconditioners.

In order to further clarify the Kernel concept, we provide a simple example (single phase water pressure diffusion) kernel here. The diffusion of pressure written as $\nabla \cdot\left(\frac{k \rho_{w}}{\mu_{w}} \cdot \nabla p_{w}\right)$ equation, which contributes to the overall residual in the system is provided as an example. Figure 3 shows the actual codes of the pressure diffusion kernel. In this figure, _test is the test function evaluated at the quadrature point_qp and_grad_phi is the gradient of shape function evaluated at the quadrature point _qp (both provided by MOOSE), _u and _grad_u are the current solution variable and the gradient of the current solution variable this kernel operates on evaluated at the quadrature point _qp. permeability is the intrinsic material permeability defined in material kernel that the physics kernels can access, _dens_water and _visc_water are the water phase fluid density and viscosity, respectively, as returned from the equation of state auxiliary Kernel. Every other term based upon the MOOSE framework, the FALCON code has developed a set of 'physics' kernels handling the time derivatives, single- and two-phase flow equations, heat and energy transport, source-sink terms, geomechanics, as well as a set of 'Auxiliary' and 'Material' kernels for equations of state (EOS) and flow-transport-mechanical properties required for geothermal reservoir simulations. As shown and discussed in the sections that follow, these kernels all have modular, pluggable structure, and can be coupled in arbitrary ways depending on the type of problems of interest. It is also worth noting that the MOOSE framework provides a material kernel. Flow and transport properties such as porosity, permeability, and relative permeability can all be defined within this material kernel and can be accessed by the physics kernels during each residual evaluation. Furthermore, the material kernel has access to state variables if needed. This feature is particularly useful for hydrofracturing applications where fracturing significantly modifies the porosity and permeability of porous media. 


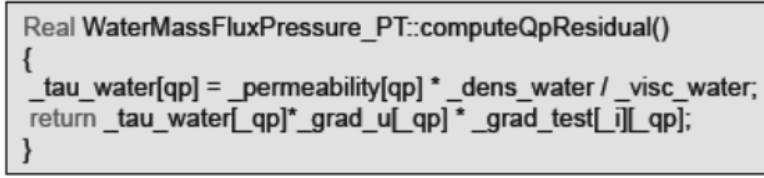

Figure 3: Residual (left) and Jacobian (preconditioner, right) evaluations inside the pressure diffusion kernel for single phase flow of water.

\section{Example Applications}

\subsection{Comparison with analytical solution}

Our first example problem is to solve a simple onedimensional heat conduction-convection problem using FALCON and compare the numerical solution with the analytical solution. In this particular example, only two equations, fluid flow and heat transport, are solved.

The analytical solution compared in this example is derived from the solution by Faust and Mercer [5], by omitting the heat exchange between confined aquifer and surrounding rock matrix. In order to obtain the analytical solution, the thermodynamic and transport properties, such as water density and viscosity are assigned as constants. Then the mass conservation equation reduced to a Laplacian equation of pressure (Equation 1 ), which gives a uniform velocity $v_{w}$ along $x$ direction.

$$
\tau \nabla^{2} P+q^{\prime}{ }_{w}=0
$$

And the energy equation reads as:

$$
K_{m} \frac{\partial^{2} u}{\partial x^{2}}-v_{w} \rho_{w} c_{w} \frac{\partial u}{\partial x}=\rho_{m} c_{m} \frac{\partial u}{\partial t}
$$

where $\rho_{m} c_{m}=\varphi \rho_{w} c_{w}+(1-\varphi) \rho_{r} c_{r}$. The $c$ is specific heat capacity of water (subscripted with $w$ ) or rock (subscripted with $r$ ). And $u$ is normalized temeperature $u=\frac{T-T_{0}}{T_{i}-t_{0}}, T_{i}$ and $T_{0}$ are the injection and initial temperature, respectively. $K_{m}$ is the heat conductivity of wet rock.

The analytical solution for Equivation (1) and (2) is given by Avdonin [1]:

$$
\begin{array}{r}
u(\chi, \tau)=\frac{\chi}{(\pi \tau)^{1 / 2}} \int_{0}^{1} \exp \left[-\left(s \gamma(\tau)^{\frac{1}{2}}\right.\right. \\
\left.\left.-\frac{\chi}{2 s(\tau)^{1 / 2}}\right)^{2}\right] \frac{d s}{s^{2}}
\end{array}
$$

where $\chi=\frac{2 x}{b}, \tau=\frac{4 K_{m} t}{c_{m} \rho_{m} b^{2}}, \gamma=\frac{Q c_{w} \rho_{w}}{4 K_{m}}, Q$ is the injection rate, and $b$ is the reservoir thickness $(1-\mathrm{m}$ in this example).

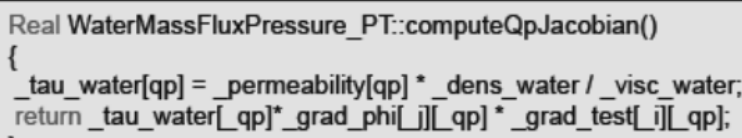

\begin{tabular}{lll}
\hline Parameter & Value & Units \\
\hline Porosity & 0.20 & - \\
\hline Permeability & $1 \times 10^{-15}$ & $\mathrm{~m}^{2}$ \\
\hline Rock Density & $2.5 \times 10^{3}$ & $\mathrm{~kg} / \mathrm{m}^{3}$ \\
\hline Rock Specific Heat & $0.92 \times 10^{3}$ & $\mathrm{~J} / \mathrm{kg}^{\circ} \mathrm{C}$ \\
\hline Thermal Conductivity & 1.5 & $\mathrm{~W} / \mathrm{m}^{\circ} \mathrm{C}$ \\
\hline Water Density & $1 \times 10^{3}$ & $\mathrm{~kg} / \mathrm{m}^{3}$ \\
\hline Water Specific Heat & $4.186 \times 10^{3}$ & $\mathrm{~J} / \mathrm{kg}^{\circ} \mathrm{C}$ \\
\hline
\end{tabular}

Table 1: Parameters used for the 1-dimensional convection-conduction problem numerical-analytical comparison.

In FALCON simulations, the geometry used for this example is a 100 meter long rectangle, 1 meter in width, with a 1 meter grid resolution. The mesh consisted of 100 elements and 102 nodes. Table 1 summarizes the parameters used for this example. Initial conditions are set as pressure $P=10 M P a$, temperature $T=$ $200^{\circ} \mathrm{C}$, uniformly. The $\mathrm{BC}$ are set as: injection pressure $P_{i}=10.5 \mathrm{MPa}$, temperature $T i=150^{\circ} \mathrm{C}$ at left side, and constant pressure $10 \mathrm{MPa}$, temperature $200^{\circ} \mathrm{C}$ are assigned at right side.

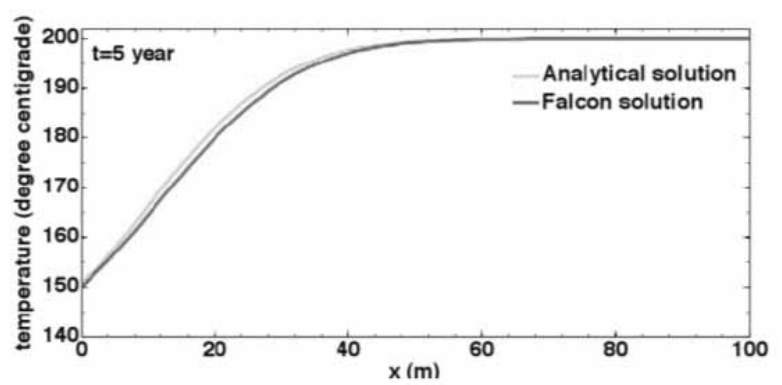

Figure 4: Comparison of the numerical and analytical solutions for 1-dimensional heatconductionconvection problem. Temperature profile calculated by FALCON and analytical solution at 5 years. The small discrepancy is caused by the pressure and temperature dependent density and viscosity of water used in the FALCON simulations. The analytical solution assumes a constant uid density and viscosity, which essentially decouplesthe the flow and transport problem. 
Figure 4 shows the comparison between numerical and analytical solutions after 5 years of simulated transport. It is clear that the numerical solution agrees well with the analytical solution. The small discrepancy is caused by the pressure and temperature dependent density and viscosity of water used in the FALCON simulations. The analytical solution assumes a constant fluid density and viscosity, which essentially decouples the flow and transport problem.

\subsection{Thermal stimulation of a geothermal reservoir}

Management of fluid reinjection is of critical importance for maintaining geothermal reservoir performance. Reinjection has posed a problem for portions of the Hellisheidi Geothermal Field, southwest Iceland, where a number of wells are drilled into active faults. The Hellisheidi Geothermal Field is located in the southern part of the Hengill Area, an active volcanic system consisting of Mt. Hengill and fracture/fault zones to the north- and south-west (Smundsson, 1967; Franzson et al., 2005). Injection tests have resulted in swarms of small earthquakes and with the injectivity of the wells exhibiting a high dependence on temperature of the reinjected water. Strongly coupled thermo- hydromechanical effects on fractures in the fracture-governed reservoir likely explain the temperature dependent injectivity.

A number of injectivity tests have been conducted to support the development of the Hellisheidi Power Plant. For several of the wells, injection experiments were conducted using three types of water; $120^{\circ} \mathrm{C}$ untreated brine directly from the low-pressure boiler, a $90^{\circ} \mathrm{C}$ mixture of brine and condense water (7:3) from the turbines, and $15^{\circ} \mathrm{C}$ cold groundwater. These experiments were done in the three most promising wells in the Hsmli Reinjection Zone, HN-09, HN-12, and HN-16. The injectivity vs. $T$ is plotted for all the wells in Figure 5. It should be mentioned here that the values for cold water in wells $\mathrm{HN}-12$ and HN-16 are inaccurate. The wells are so permeable that the pressure changes in the pumping tests were not very clear.

The injection tests for estimating the injectivity were conducted as described below. Maximum flow of water at preferred temperature was injected into the well for several days. The wellhead pressure was monitored in order to estimate when the well had reached equilibrium.
A pressure and temperature sensor was placed in the well at the depth of its main feed zone. The flow was lowered in three steps, each lasting for approximately 3 hours to allow the pressure to equilibrate to the new injection rate. Figure 6 is an example of pumping test results for the hot water injection into well HN-09. The pressure and temperature are plotted over the duration of the injection steps.

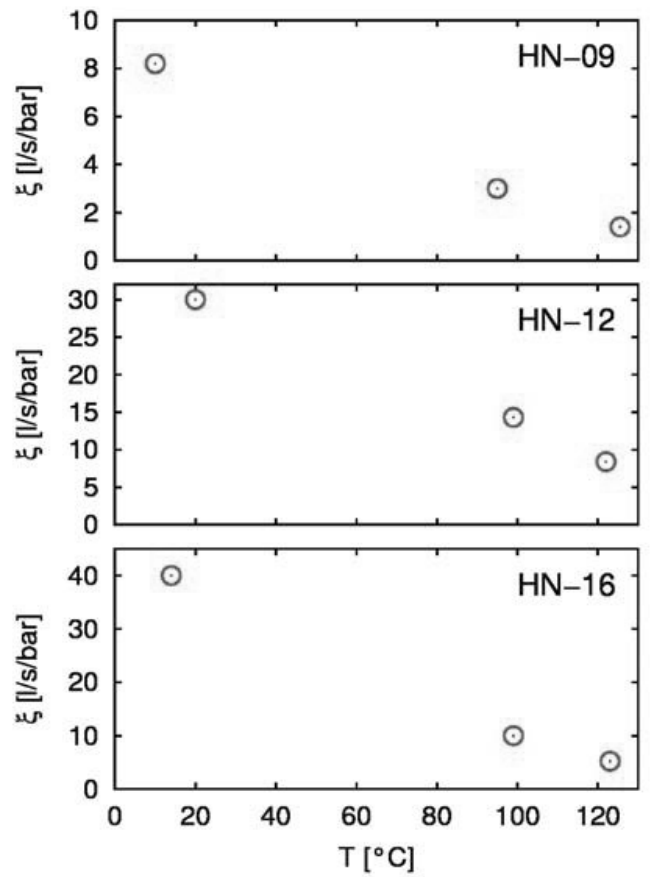

Figure 5: Injectivity at different values of temperature (T) in three wells in the Hsmli Reinjection Zone. The injectivity values for the lowest temperatures in wells $\mathrm{HN}-12$ and $\mathrm{HN}-16$ are not very accurate.

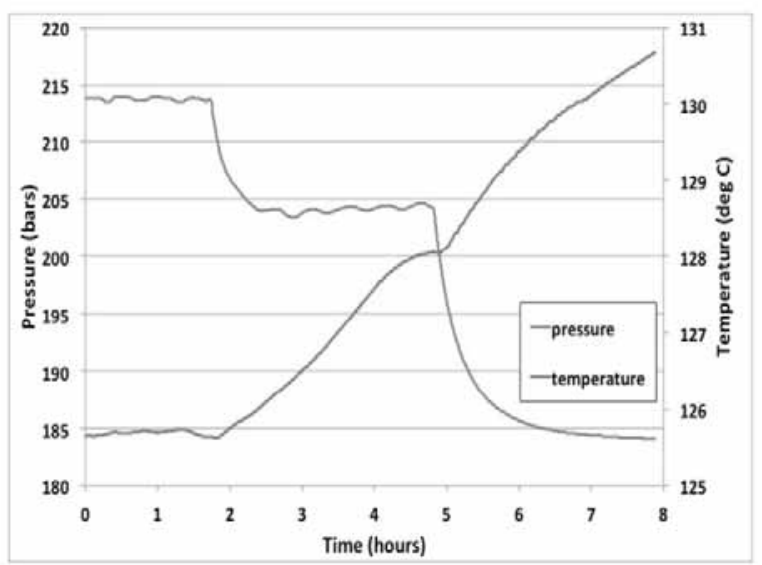

Figure 6: Injection test in well HN-09 using 120 C water. The pressure sensor is placed $30 \mathrm{~m}$ above the bottom of the well showing the pressure and temperature over the dura- tion of the test. 


\section{Model Setup}

A radial structured mesh, with a radius of 250 meters, is being used to simulate the injection into well $\mathrm{HN}-09$. The simulation domain is 100 meters thick, with the production zone begin represented as a 5 -meter thick zone of fractured rock embedded in lower permeability (unfractured) reservoir rock. Figure 7 shows the simulation domain and the computational mesh.

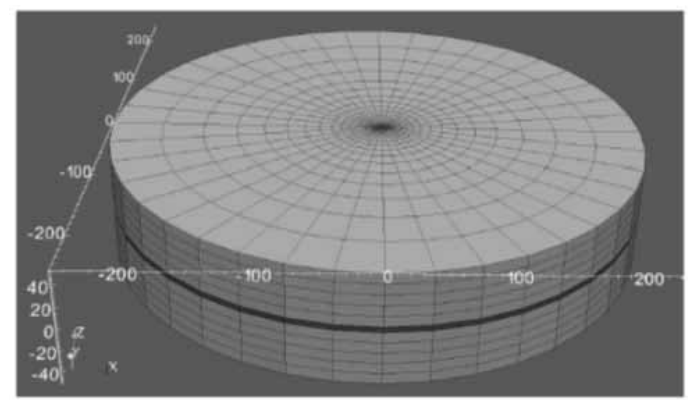

Figure 7: Computational domain used for the numerical simulations of injection into well HN-09. The red zone in the middle of the domain represents the fracture zone/fault system comprising the feed zone of the well.

Initial conditions being used in the simulations mimic those that exist in the reservoirs. The initial temperature distribution used in the simulation is shown in Figure 8, with the resulting water density, as calculated from the IAPWS-97 steam tables, shown on Figure 9. The temperature in the feed zone is approximately $264^{\circ} \mathrm{C}$, with the temper- ature ranging from $263^{\circ} \mathrm{C}$ to $265^{\circ} \mathrm{C}$. The water density in the reservoir ranged from approximately 772 to $776 \mathrm{~kg} / \mathrm{m}^{3}$ in the initial conditions.

The initial pressure in the reservoir was specified to a uniform 185 bars, and as the majority of the flow was expected to be primarily horizontal and limited to exist only within the thin fracture zone, the effects of gravity of the fluid flow and heat transport were neglected. This approach greatly simplified the specification of the boundary conditions needed for the simulations.

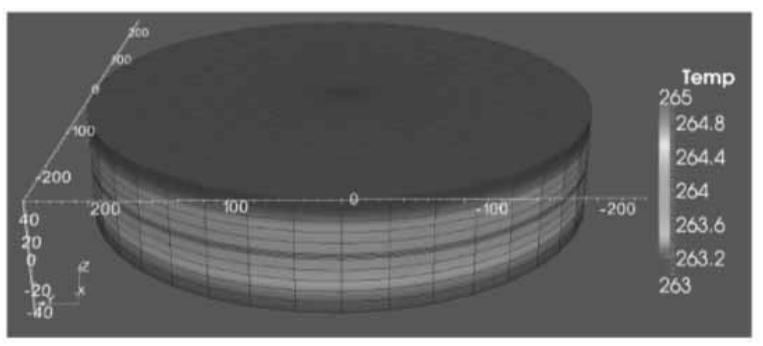

Figure 8: Initial temperature used for the simulations.

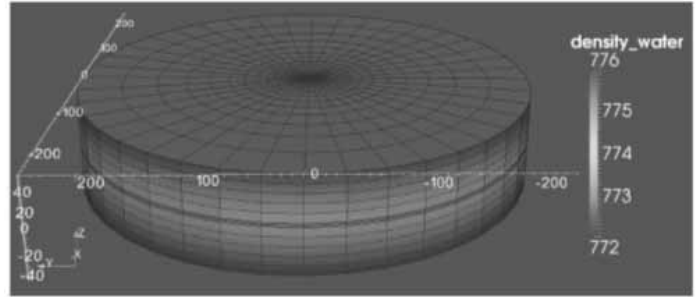

Figure 9: Initial water density distributions used for the simulations.

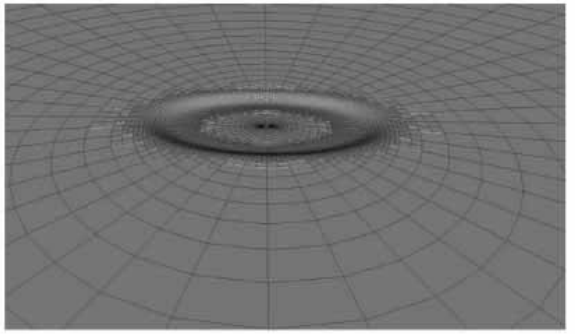

Figure 10: Predicted thermal contraction of the reservoir matrix in the feed zone in the vicinity of well $\mathrm{HN}-09$. Note that the deformation is greatly exaggerated for illustration purposes.

Feedback between the geomechanics and fluid flow are being implemented by revising the permeability of the feed zone by an effective fracture aperture, as calculated by the thermo-mechanical deformation of the mesh resulting from the injection of cold fluid into the initially hot reservoir. As the host reservoir rock thermally contracts, the effective permeability from the fractured feed zone increases using a cubic law approxi- mation.

An initial modeling scenario, consisting of injecting approximately $30 \mathrm{l} / \mathrm{s}$ of $20^{\circ} \mathrm{C}$ pure water into the approximately $260^{\circ} \mathrm{C}$ reservoir, is currently underway. Preliminary simulation results are encouraging, where permeability increases on the order of $10 X$ to $100 X$ are predicted in the vicinity of well HN-09's feed zone. The reservoir matrix contraction in the feed zone near the injection well is predicted to approach $10^{-4}$ meters.

Figure 10 shows the results of the geomechanical deformation in the vicinity of the injection well in the feed zone, along with the mesh that is adaptively refined by FALCON to capture the strong gradient in the temperature and resulting thermal deformation in the reservoir.

\section{Acknowledgment}

The author would like to acknowledge travel support to the SIMS Conference provided by the International Energy Agency's Geothermal Implementing Agreement (IEA-GIA). 
This contribution is a post-conference publication from SIMS 2012 Conference (53rd SIMS Conference, Reykjavík, Iceland, October 4 - 6, 2012). The contribution is a (partly) modified publication from the paper published in the Proceedings of SIMS 2012, published by Orkustofnun, National Energy Authority Iceland, ISBN: 978-9979-68-318-6, electronically available at http://www.scansims.org/sims2012/SIMS2012.pdf.

\section{References}

[1] Avdonin, NA. Some formulas for calculating the temperature of a stratum subject to thermal injection. Izvestiya Vysshikh Uchebnykh Zavedenii. 1964; 7(4):37-41.

[2] Balay S, Buschelman K, Eijkhout V, Gropp WD, Kaushik D, Knepley MG, McInnes LC, Smith BF, Zhang H. PETSc users manual. Technical Report ANL95/11 - Revision 2.1.5, Argonne National Laboratory, 2004.

[3] Carrayrou J, Knabner P, Hoffmann J, Krautle S, de Dieuleveult C, Erhel J, van der Lee J, Lagneau V, Mayer KU, MacQuarrie KTB. Comparison of numerical methods for simulating strongly nonlinear and heterogeneous reac- tive transport problems-the momas benchmark case. Computational Geosciences. 2010; 14(3):483-502.

[4] Falgout RD, Yang UM. hypre: A library of high performance preconditioners. In Computational Science-Iccs 2002, Berlin, 2002. Springer-Verlag.

[5] Faust CR, Mercer JW. Geothermal reservoir simulation .1. Mathematcial- models for liquid-dominated and vapot-dominated hydrothermal systems. Water Resources Research. 1979; 15(1):23-30.

[6] Gaston D, Newman C, Hansen G, Lebrun-Grandié D. MOOSE: A parallel computational framework for coupled systems of nonlinear equations. Nuclear Engineering and Design. 2009; 239: 1768-1778.

[7] Guo L, Huang H, Gaston D, Permann C, Andrs D, Redden G, Lu C, Fox D, Fujita Y. A parallel fully-coupled fully-implicit solution to reactive transport in porous media using preconditioned jacobian-free newton-krylov method. Journal of Computational Physics, in review.

[8] Hammond GE, Valocchi AJ, Lichtner PC. Application of jacobian-free newton-krylov with physics-based preconditioning to biogeochemical transport. Advances in $\mathrm{Wa}$ ter Resources. 2005; 28(4):359-376.

[9] Heroux M et al. Trilinos: an object-oriented software framework for the solution of large-scale, complex multi-physics engineering and scientific problems. http://trilinos.sandia.gov, 2008.

[10] Kirk BS, Peterson JW, Stogner RH, Carey GF. libMesh: a $\mathrm{C}++$ library for parallel adaptive mesh refinement/coarsening simulations. Eng Comput-Germany. 2006; 22(3-4): 237-254.
[11] Knoll D, Park R, Smith K. Application of the Jacobianfree Newton-Krylov method in computational reactor physics. In American Nuclear Society 2009 International Conference on Advances in Mathematics, Computational Methods, and Reactor Physics, May 2009; Saratoga Springs.

[12] McHugh PR, Knoll DA. Inexact Newton's method solutions to the incompressible Navier-Stokes and energy equations using standard and matrix-free implementations. AIAA J. 1994; 32: 2394.

[13] Miller CW, Benson LV. Simulation of solute transport in a chemically reactive heterogeneous system - model development and application. Water Resources Research. 1983; 19(2): 381-391.

[14] Molins S, Carrera J, Ayora C, Saaltink MW. A formulation for decoupling components in reactive transport problems. Water Resources Research. 2004; 40(10).

[15] Rutqvist J, Wu YS, Tsang CF, Bodvarsson G. A modeling approach for analysis of coupled multiphase fluid flow, heat transfer, and deformation in fractured porous rock. Int. Journal of Rock Mechanics and Mining Sci. 2002; 39(4): 429-442.

[16] Steefel CI, MacQuarrie KTB. Approaches to modeling of reactive transport in porous media. Reviews in Mineralogy and Geochemistry, 1996; 34: 83-129.

[17] Valocchi AJ, Street RL, Roberts PV. Transport of ionexchanging solutes in groundwater - chromatographic theory and field simulation. Water Resources Research. 1981; 17(5):1517-1527.

[18] Wagner W, Cooper JR, Dittman A, Kijima J, Kretzschmar HJ, Kruse A, Mares R, Oguchi K, Sato H, Stocker I, Sifner O, Takaishi Y, Tanishita I, Trubenbach J, Willkommen Th. The IAPWS Industrial Formulation 1997 for the Thermodynamic Properties of Water and Steam. Trans. ASME. 1997; 150(122):150-182.

[19] Xu T, Sonnenthal E, Spycher N, Pruess K. TOUGHREACT - A simulation program for non-isothermal multiphase reactive geochemical transport in variably saturated geologic media: Applications to geothermal injectivity and $\mathrm{CO} 2$ geological sequestration. Computers \& Geosciences. 2006; 32(2):145-165.

[20] Yeh GT, Sun J, Jardine PM, Burgos WD, Fang Y, Li MH, Siegel MD. HYDROGEOCHEM 5.0: A Coupled Model of Fluid Flow, Thermal Transport, and HYDROGEOCHEMical Transport through SaturatedUnsaturated Media: Version 5.0. Technical Report ORNL/TM-2004/107, Oak Ridge National Laboratory, Oak Ridge, TN, 2004.

[21] Yeh GT, Tripathi VS. A critical-evaluation of recent developments in hydrogeochemical transport models of reactive multichemical components. Water Resources Research. 1989; 25(1): 93-108. 